\title{
Dislocation-like Structures in a Simulated Liquid
}

\section{Cotterill, Rodney M J}

Published in:

Physical Review Letters

Link to article, DOI:

10.1103/PhysRevLett.42.1541

Publication date:

1979

\section{Document Version}

Publisher's PDF, also known as Version of record

Link back to DTU Orbit

\section{Citation (APA):}

Cotterill, R. M. J. (1979). Dislocation-like Structures in a Simulated Liquid. Physical Review Letters, 42(23), 1541-1544. https://doi.org/10.1103/PhysRevLett.42.1541

\section{General rights}

Copyright and moral rights for the publications made accessible in the public portal are retained by the authors and/or other copyright owners and it is a condition of accessing publications that users recognise and abide by the legal requirements associated with these rights.

- Users may download and print one copy of any publication from the public portal for the purpose of private study or research.

- You may not further distribute the material or use it for any profit-making activity or commercial gain

- You may freely distribute the URL identifying the publication in the public portal

If you believe that this document breaches copyright please contact us providing details, and we will remove access to the work immediately and investigate your claim. 


\title{
Dislocationlike Structures in a Simulated Liquid
}

\author{
R. M. J. Cotterill \\ Department of Structural Properties of Materials, The Technical University of Denmark, \\ DK-2800 Lyngby, Denmark \\ (Received 1 March 1979)
}

The free-volume distribution in a simulated Lennard-Jones liquid is heterogeneous. Chains of holes, appearing as segments rather than a continuous network, have lifetimes that are brief compared with the mean vibration period of the atoms. Larger isolated holes persist for longer times. If the chains are interpreted as dislocation cores, the observed dislocation density is $1.0 \times 10^{14} \mathrm{~cm}^{-2}$, but the actual density is probably higher.

The melting transition has become the object of considerable attention. ${ }^{1-4}$ Experimental methods have emerged for studying the melting of twodimensional (2D) systems, ${ }^{5,6}$ and theoretical advances have endorsed the view that melting proceeds via a sudden proliferation of dislocations. This was first observed in a 2D computer simulation, ${ }^{7}$ while a subsequent analysis by Kosterlitz and Thouless ${ }^{8,9}$ generalized the dislocation model to include magnetic and superfluid transitions in two dimensions. The development of these ideas ${ }^{10,11}$ led to predictions of the effect of analogous excitations (vortices) on the superfluid density in a ${ }^{4} \mathrm{He}$ film, and experimental verification ${ }^{12,13}$ provided further implicit support for the dislocation theory. Progress with 3D systems has been slower, but here too there is evidence, from computer simulation ${ }^{14}$ and electron microscopy, ${ }^{15}$ that dislocations are involved in melting.

A two-phase dislocation theory of melting implies acceptance of the view that a liquid can be described in terms of dislocations, and Shockley's calculation ${ }^{16}$ of liquid viscosity was a notable success for this approach. Interpretations of how the defect is to be incorporated into a liquid model range from the Mott-Gurney picture ${ }^{17}$ of a microcrystalline array, and the dislocation-saturated crystal as variously advocated by Ookawa, ${ }^{18} \mathrm{Miz}-$ ushima, ${ }^{19} \mathrm{Siol},{ }^{20}$ and Kuhlmann-Wilsdorf, ${ }^{21}$ to the more nebulous idea that in a liquid only the dislocation cores survive. ${ }^{22}$ A dislocation core is a region of dilatation, and this facilitates atomic motion because of the local increase in interatomic distances and hence lowering of energy barriers. In a liquid such cores would be expected to produce a network of locally dilated regions, the individual segments appearing as chains of holes. This communication reports the first observation of such structures in a simulated liquid.

The Lennard-Jones liquid, simulated by molecular dynamics, comprised 336 atoms in the ir- reducible cell of a system made pseudoinfinite by periodic boundary conditions. Reduced variables were employed for computational efficiency. ${ }^{14}$ The energy and distance parameters, $\epsilon$ and $\sigma$, in the interaction potential $V(r)=\epsilon\left[(\sigma / r)^{12}-2(\sigma / r)^{6}\right]$ were set at unity. With mass and time, and Boltzmann's constant, similarly reduced, the melting temperature was $T=0.72$. The liquid was equilibrated for ten thousand computation cycles at $T=0.76$ and analyzed at various times during a continuation of the simulation at constant temperature and pressure.

The analysis consisted of constructing a threedimensional grid of test points, filling the irreducible cell, with a mesh size of 0.05 atomic diameters, i.e., several thousand test points per atom. If a test point lay inside an atom, it was rejected. For all the remaining test points the shortest distance to the surface of the nearest atom was computed, the atoms being regarded as hard spheres with diameter equal to the distance of the first peak of the pair distribution function. This produced a distribution of spherical test holes with an upper cutoff radius of 0.30 . (The vacancy radius in the equivalent close-packed crystal at zero temperature would be 0.50 .) The complete set of test holes naturally displayed considerable interpenetration because of the small mesh size, and graphical display of every hole would have been confusing. Instead, the holes were arranged in decreasing order of size and plotted sequentially in that order. Moreover, before any hole was plotted, its position was checked against every larger hole already plotted, and rejected if the distance between it and the nearest plotted hole was less than half the sum of their radii. The plotting was terminated at a hole radius of 0.19 . This is $10 \%$ less than the radius of the largest spherical hole in the corresponding close-packed crystal at zero temperature: the inscribed sphere of the octahedral cavity (radius $=0.21$ ) in the face-centered cubic 
structure. The extra $10 \%$ allowed for the vibrational amplitude at the melting point, as given by the Lindemann rule. ${ }^{23}$ To facilitate 3D display, all the retained test holes were plotted in stereo pairs.

Figure 1(a) shows such a stereo pair, the rectangular box indicating the periodic boundaries. With a stereo viewer in correct adjustment the legends appear to lie in the median plane. To reduce plotting time, a standard octagonal symbol was used, the circumscribed circle of which has a radius equal to that of the test hole in question. It was found that chains of test holes could be detected by eye, and these were drawn in on the original plots as lines, which were in general slightly curved. For clarity, the lines corresponding to Fig. 1(a) are presented on a separate stereo pair in Fig. 1(b). The observed chains varied in length, the longest comprising seven holes and almost spanning the width of the irreducible cell. Figures 2(a) and 2(b) are similar plots corresponding to a time ten computational time steps after Fig. 1, and Figs. 3(a) and 3(b) show the situation after a further ten steps. (The mean atomic vibration period in the corresponding crystal was approximately fifty computational time steps, i.e., about $10^{-12} \mathrm{sec}$ ). Several points
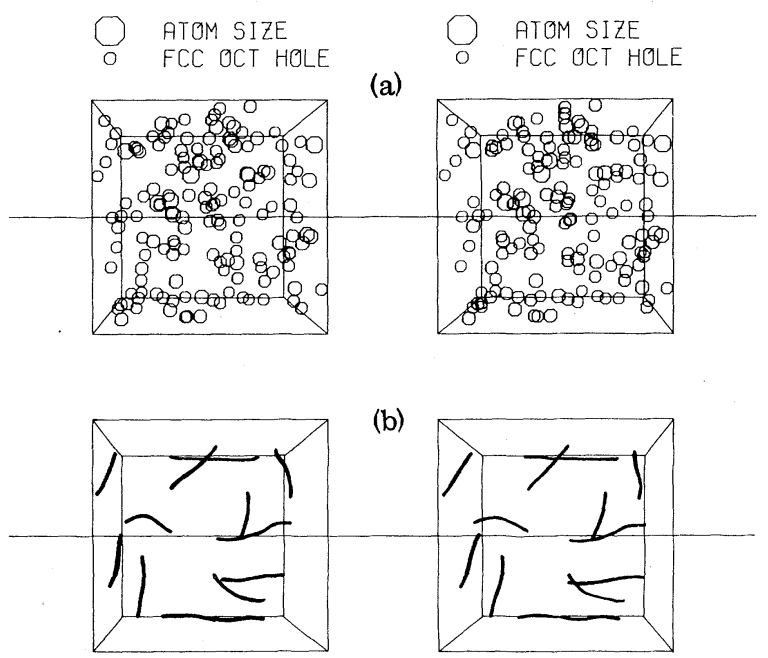

FIG. 1. (a) Stereo view of the distribution of test holes having a radius larger than 0.19 , at one instant during the simulation of the atomic motions in a liquid. The legends, which should appear to lie in the median plane of the figure, indicate the diameters of an atom and the inscribed sphere of the octahedral hole in the fcc structure. The box marks the periodic boundaries. (b) Stereo view of the positions of well-defined chains of holes seen in (a). Note that they appear as isolated segments rather than as a continuous network. emerged from a study of many plots of this type. The largest holes are not the most mobile, in contradistinction to what is expected if a vacancytype motion is operative; these holes are relatively stationary and undergo a sort of breathing mode due to the vibrations of surrounding atoms. Some small holes pop into and out of view at isolated locations, probably a result of size fluctuations of holes whose mean size is below the cutoff value. The main observation is the heterogeneity of the spatial distribution of the holes. This is apparent from a cursory inspection of Figs. 1(a), 2(a), and 3(a), while 3D viewing reveals several hole-free regions roughly three atomic diameters across. The arrangement of the chains of holes changes very rapidly, a time interval corresponding to one mean vibrational period in the crystalline state being sufficient to permit relaxation to a totally new configuration. During the vriefer time intervals between the figures, some of the chains appear to be relatively stationary while others vanish abruptly.

Two remarks are necessary regarding the significance of the claimed positional correlations of test holes. Firstly, no statistical test was applied to the actual observations, and it is not clear how this could have been carried out since the observed chains of holes were not straight lines, and did not form a continuous network. Instead, a bogus plot was produced by generating test holes with random positions and diameters (with the same size distribution as in the
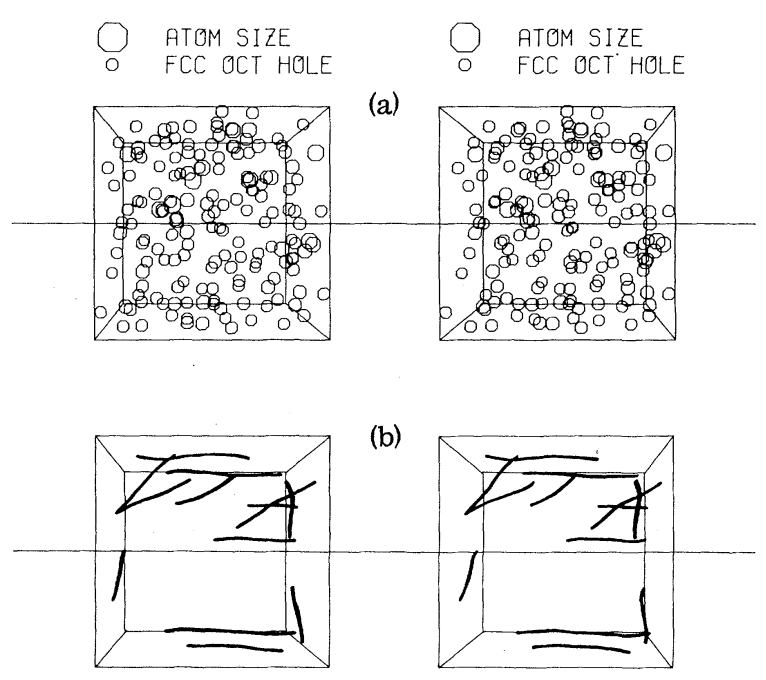

FIG. 2. (a) The distribution of test holes, ten computational time steps after Fig. 1(a). (b) The positions of well-defined chains of holes as seen in (a). 
molecular-dynamics runs). Where these holes overlapped, they were subjected to the same rejection criterion that applied to Figs. 1(a), 2(a), and 3(a). Figure 4 shows such a plot, and 3D viewing reveals no extended chains of test holes. Secondly, a continuous network of holes must be seen if the plotting limit is small enough. In the present simulations, such a network is present for a radius limit of 0.13 , but the number of network junctions is then comparable to the number of atoms, and the implied situation with every atom dislocated seems physically unreasonable.

Of the two types of defect conjecturally linked with the liquid state, the present results appear to rule out the vacancy ${ }^{24}$ as a viable candidate. The largest test hole observed during the simulation had a radius of 0.30 , which is $40 \%$ less than that of a vacancy in the close-packed crystal at zero temperature. This conflicts with the reported observation of vacancies in simulated liquid rubidium, ${ }^{25}$ but supports, admittedly indirectly, the conclusion that vacancies play no part in melting. ${ }^{26}$

If the observed chains of holes are to be interpreted as dislocation cores, the dislocation density corresponding to Figs. 1(a), 2(a), and 3(a) is approximately 0.11 per atomic area [i.e., approximately $1.0 \times 10^{14} \mathrm{~cm}^{-2}$, taking the first peak of the pair distribution function of a Lennard-Jones liquid to lie at $3.7 \AA$ (Ref. 27)]. This is of the same order of magnitude as, but somewhat smaller than, the saturation dislocation density pre-
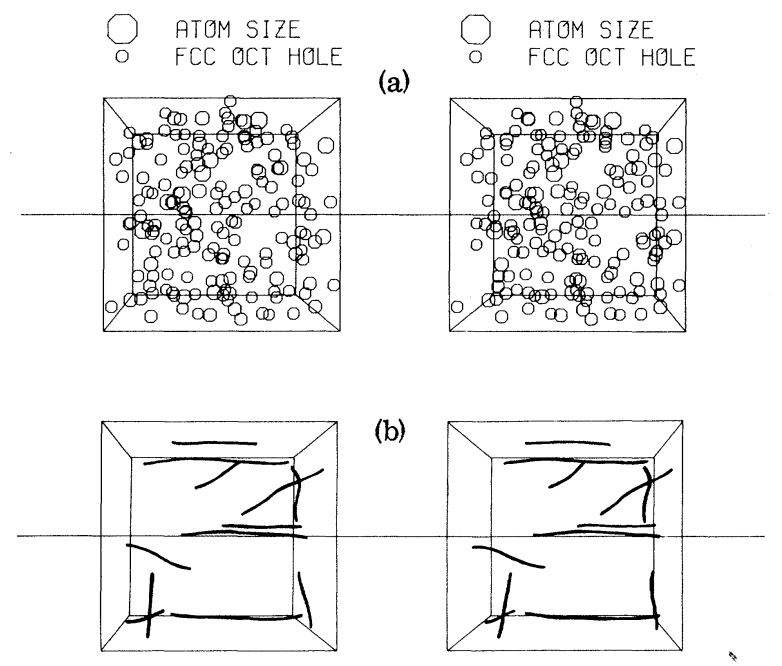

FIG. 3. (a) The distribution of test holes, ten computational time steps after Fig. 2(a). (b) The positions of well-defined chains of holes as seen in (a). dicted for the liquid state. ${ }^{22}$ To this apparent discrepancy must be added the seemingly more serious shortcoming that the present observations reveal only isolated chain segments rather than a fully connected network. Dislocations in crystals cannot end abruptly but must either link up with other dislocations or terminate at a surface. There are, however, at least two reasons why a network would not be seen in the simulated liquid. The rigorous constraint of constancy of Burgers vector, and hence of core diameter, might be relaxed in the noncrystalline state, ${ }^{28,29}$ permitting some of the associated test holes to fall below the plotting limit and thus cause an apparent break in an otherwise continuous chain. Moreover, the dislocations in a liquid must be in continuous motion.

Consider the observation of test holes at a dislocation moving in a crystal. The discrete nature of the crystal lattice forces the dislocation to make quantum jumps, of distance equal to the Burgers vector, that carry it from one minimum of potential energy to an adjacent minimum. These minima correspond to the greatest local concentration of free volume, and each gives rise to a chain of holes along the core. During a jump, the holes will disappear at one site and reappear at a site about one atomic diameter away. Identification of a dislocation by detection of a concatenation of test holes is thus possible only when the dislocation is not actually making a jump. The same is true for a liquid; similar quantum jumps would have to be made by the moving dislocation, because although the liquid state is noncrystalline, it is nevertheless composed of discrete entities. (Complications arise because the dislocation concentration in a liquid must be high, and the movement of one dislocation will alter the environments of those nearby.) The dislocation cores in a liquid might form a proper-

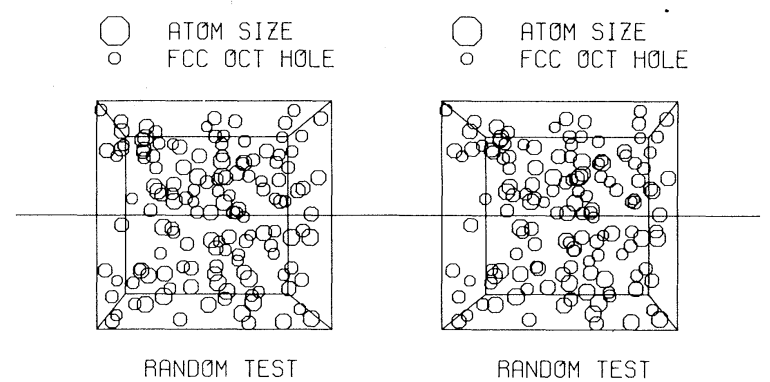

FIG. 4. The distribution of test holes generated at random. Chains of holes are not observed. 
ly connected network, but those segments which are in motion at the instant of observation will not be detectable. An evaluation of the dislocation density in the liquid, based on instantaneous observation of only a fraction of the dislocation cores, must produce an underestimate. No attempt was made to calculate what fraction of the total dislocation content is invisible at any one time, but a reliable measure of this number might enable one to estimate the height of the PeierlsNabarro barrier. ${ }^{30}$ This, in turn, would lead to a numerical value for the viscosity. It would thus appear that there is much to be gained by repeating the present study for the corresponding supercooled liquid. In that glassy state there would presumably be more dislocations visible; possibly even a continuous network.

I am grateful to Sir Nevill Mott and Sir Rudolph Peierls for stimulating comments. Discussions with Masao Doyama, Toshiyuki Ninomiya, Hideji Suzuki, Jeffery Tallon, and Tyoichi Yamamoto have been of great value, and I am indebted to Sir Sam Edwards for sending me a preprint of a paper, co-authored with M. Warner, on dislocations and melting. This work was supported by the Danish Natural Science Research Council.

${ }^{1}$ B. I. Halperin and D. R. Nelson, Phys. Rev. Lett. $\underline{41}$, 121 (1978).

${ }^{2}$ R. Clarke, N. Caswell, and S. A. Solin, Phys. Rev. Lett. $\underline{42}, 61$ (1979).

${ }^{3}$ V. L. Pokrovsky and A. L. Talapov, Phys. Rev. Lett. 42, 65 (1979).

${ }^{4}$ A. Holz and J. T. N. Medeiros, Phys. Rev. B $\underline{17}$, 1161 (1978).

${ }^{5}$ J. G. Dash, Films on Solid Surfaces (Academic, New York, 1975).

${ }^{6} \mathrm{H}$. Shechter, J. Suzanne, and J. G. Dash, Phys. Rev. Lett. 37, 706 (1976).

${ }^{7}$ R. M. J. Cotterill and L. B. Pedersen, Solid State Commun. 10, 439 (1972).
${ }^{8}$ J. M. Kosterlitz and D. J. Thouless, J. Phys. C $\underline{5}$, L124 (1972).

${ }^{9}$ J. M. Kosterlitz and D. J. Thouless, J. Phys. C $\underline{6}$, 1181 (1973).

${ }^{10}$ J. M. Kosterlitz, J. Phys. C 7, 1046 (1974).

${ }^{11}$ D. R. Nelson and J. M. Kosterlitz, Phys. Rev. Lett. 39, 1201 (1977).

${ }^{12}$ D. J. Bishop and J. D. Reppy, Phys. Rev. Lett. 40, 1727 (1978); see also V. Ambegaokar, B. I. Halperin, D. R. Nelson, and E. D. Siggia, Phys. Rev. Lett. $\underline{40}$, 783 (1978).

${ }^{13}$ I. Rudnick, Phys. Rev. Lett. 40, 1454 (1978).

${ }^{14}$ R. M. J. Cotterill, E. J. Jensen, and W. Damgaard Kristensen, Philos. Mag. 30, 245 (1974).

${ }^{15}$ R. M. J. Cotterill and J. Klæstrup Kristensen, Philos. Mag. 36, 453 (1977). .

${ }^{16} \mathrm{~W}$. Shockley, l'Etat Solide (Institut International de Physique Solvay, Brussels, 1952), p. 431.

${ }^{17}$ N. F. Mott and R. W. Gurney, Trans. Faraday Soc. 35, 364 (1939); see also J. K. MacKenzie and N. F. Mott, Proc. Phys. Soc., London 63, 411 (1950); N. F. Mott, Proc. Roy. Soc. London, Ser. A 215, 1 (1952).

${ }^{18}$ A. Ookawa, J. Phys. Soc. Jpn. 15, 2191 (1960).

${ }^{19}$ S. Mizushima, J. Phys. Soc. Jpn. 15, 70 (1960). ${ }^{20}$ M. Siol, Z. Phys. 164, 93 (1961).

${ }^{21}$ D. Kuhlmann-Wilsdorf, Phys. Rev. 140, A1599 (1965).

${ }^{22}$ R. M. J. Cotterill, E. J. Jensen, W. Damgaard Kristensen, R. Paetsch, and P. O. Esbjørn, J. Phys.

(Paris), Colloq.36, C2-35 (1975); in this paper a distinction was made between normal dislocation cores and those existing in situations in which there is no noncore material left, the latter being called "pseudodislocations." The defects reported in this communication are believed to be such pseudodislocations.

${ }^{23}$ F. A. Lindemann, Z. Phys. 11, 609 (1910).

${ }^{24}$ D. E. O’Reilly, Phys. Rev. A 15, 1198 (1977).

${ }^{25}$ E. Ferm and D. E. O’Reilly, J. Phys. C $\underline{10}$, L499 (1977).

${ }^{26}$ C. L. Reynolds and P. R. Couchman, Scr. Metall. 10,605 (1976).

${ }^{27}$ A. Rahman, Phys. Rev. 136, A405 (1964).

${ }^{28}$ M. F. Ashby and J. Logan, Scr. Metall. 7, 513 (1973).

${ }^{29}$ J. J. Gilman, J. Appl. Phys. 44, 675 (19)33).

${ }^{30}$ F. R. N. Nabarro, Theory of Crystal Dislocations

(Clarendon, Oxford, 1967), p. 175. 